\title{
Jacob's Ladder as Sketched by Escher: Assessing the Performance of Broadly-Used Density Functionals on Transition Metal Surface Properties
}

Lorena Vega, Judit Ruvireta, Francesc Viñes ${ }^{*}$, Francesc Illas

Departament de Ciència de Materials i Química Física \& Institut de Química Teòrica i Computacional (IQTCUB), Universitat de Barcelona, c/Martí i Franquès 1, Barcelona, 08028, Spain.

\begin{abstract}
The present work surveys the performance of various widely-used density functional theory (DFT) exchange-correlation (xc) functionals in describing surface observable properties of a total of 27 transition metals with face-centered cubic (fcc), body-centered cubic (bcc), or hexagonal close-packed (hcp) crystallographic structures. A total of 81 low Miller index surfaces were considered employing slab models. Exemplary xc functionals within the three first rungs of Jacob's ladder were considered including the Vosko-Wilk-Nussair (VWN) xc within the local density approximation (LDA), the Perdew-Burke-Ernzerhof (PBE) within the generalized gradient approximation (GGA), and the Tao-Perdew-Staroverov-Scuseria (TPSS) as a metaGGA. Hybrids were excluded in the survey as known to fail in properly describing metallic systems. In addition, two variants of PBE were considered, the PBE adapted for solids (PBEsol), and the revised PBE (RPBE) aimed at improving adsorption energies. Interlayer atomic distances, surface energies, and surface work functions are chosen as the scrutinized properties. A comparison to available experimental data including single-crystal and polycrystalline values shows that no $\mathrm{xc}$ is best at describing all the surface properties, although in statistical mean terms the PBEsol xc functional is advised, yet PBE is recommended when considering both bulk and surface properties. Based on the present results a discussion on adapting GGA functionals to the treatment of metallic surfaces in an alternative way to metaGGA or hybrids is provided.

*Corresponding author: francesc.vines@ub.edu
\end{abstract}




\section{Introduction}

Nowadays, density functional theory (DFT) ${ }^{1}$ has become the working horse in the theoretical and computational study of chemical processes, encompassing a rich and varied range of scientific fields, including quantum chemistry, solid state physics, materials science, and heterogeneous catalysis, to name a few. This success arises mainly from the ability of DFT methods to provide relatively accurate results at an affordable computational cost. However, a general exchange-correlation (xc) functional that permits the exact calculation of the ground state energy of a given system is still missing. Over the last decades a handful of different approximations with increasing complexity and alleged accuracy have arisen trying to solve this issue. This is often conceptualized in terms of the so-called Jacob's ladder of xc systematic improvement, a.k.a. Perdew's dream. ${ }^{2}$ To a large extent such a systematic improvement very much relies on validation against experiments through appropriate atomic and molecular datasets. It has been recently found that many basic physicochemical properties are highly dependent on the employed exchange-correlation functional, ${ }^{3}$ and, concerning materials science, there are functionals better suited for particular materials families and/or properties; e.g. hybrid xc functionals are advised when dealing with light atoms molecular systems, ${ }^{4,5}$ or when treating semiconductor or insulator band structures, ${ }^{6-8}$ even if energy related properties of the latter can be already satisfactorily described within the generalized gradient approximation (GGA). ${ }^{9,10}$

Here we focus the attention on the series of transition metals (TM), as these are present in many fields of chemistry, physics, and material science, such as in nanotechnology, ${ }^{11}$ sensors, ${ }^{12}$ homogeneous ${ }^{13}$ and heterogeneous catalysis, ${ }^{14,15}$ and $\mathrm{H}_{2}$ generation in green chemistry, ${ }^{16}$ to cite some. In many of these applications, such as in heterogeneous catalysis, TMs are typically employed in the shape of nanoparticles supported on an appropriate substrate. ${ }^{17}$ Improvements related to the higher rungs of Jacob's ladder, which imply hybrid functionals, do not necessarily work for transition metals. Indeed, the optimal percentage of Hartree-Fock (HF) exchange in a hybrid xc functional required to make it suitable for bulk transition metals is close to zero. ${ }^{18}$ Therefore, apparently, the inclusion of HF exchange is detrimental for the accuracy of xc functionals on transition metals. This has been confirmed in recent studies exploring the ability of 15 different $\mathrm{xc}$ functionals in describing energetic, structural, and compressive parameters properties of bulk TMs. ${ }^{19,20}$ These studies showed that for bulk TMs, hybrid functionals perform worse than LDA, GGA, or metaGGA functionals. The presence of HF exchange in the xc functional 
leads to unphysically localized TM bands disrupting the proper description of transition metal properties. ${ }^{21}$ Furthermore, a very recent study shows that it is possible to describe the thermochemistry of $3 d$ TMs without having to rely on hybrids approaches. ${ }^{22}$

The above mentioned previous studies pointed to xc functionals within GGA approximation as the best choice for describing TM bulk properties. In particular, on average the Perdew-Burke-Ernzerhof $(\mathrm{PBE})^{23} \mathrm{xc}$ was found to be the most accurate functional while the Tao-Perdew-Staroverov-Scuseria (TPSS) ${ }^{24}$ would be the next best suited and broadly used from another Jacob's ladder rung, often considered necessary when dealing with main group element molecular systems, as is the case for adsorbates on surfaces. ${ }^{25}$ Note in passing by that there have been recent improvements on semilocal metaGGAs, ${ }^{26}$ including intermediate-range description of dispersive forces, which, in principle, can lead to a significantly description upgrading on a diversity of chemical systems. ${ }^{27}$ Finally, the VoskoWilk-Nussair parameterization of LDA would be the best adapted in that rung. ${ }^{28}$ Notice, however, that this analysis was carried out solely for bulk transition metals, where surface peculiarities were disregarded. At this point one may wonder up to which point functionals well suited in describing bulk TMs perform are equally well in describing surfaces and related properties. This is a pertinent question since xc functionals are mostly validated against bulk crystallographic structures. The answer to this question one can shed light on the adequacy of xc functionals in the treatment of surface states and related properties, an important issue in computational heterogeneous catalysis.

To clarify this issue a wide database is selected involving 27 TMs either displaying hexagonal close-packed (hcp) crystallographic structure (Sc, Y, Ti, Zr, Hf, Tc, Re, Ru, Os, $\mathrm{Co}, \mathrm{Zn}$, and $\mathrm{Cd})$, face-centered cubic $(f c c)$ one ( $\mathrm{Rh}, \mathrm{Ir}, \mathrm{Ni}, \mathrm{Pd}, \mathrm{Pt}, \mathrm{Cu}, \mathrm{Ag}$, and $\mathrm{Au}$ ), or a bodycentered cubic $(b c c)$ crystal packing (V, Nb, Ta, Cr, Mo, W, and Fe). For these $27 \mathrm{TMs}$, different low-index Miller surfaces with maximum index order of 1 have been considered, thus featuring in principle the most stable surface termination according to their crystal structure. Specifically, these are the (001), the (011), and (111) surfaces for $f c c$ and $b c c$

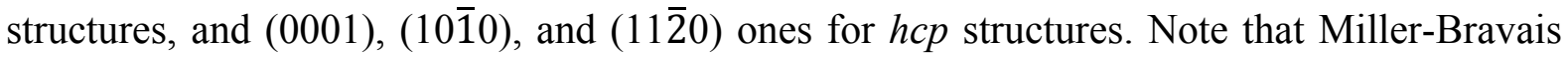
indexes are used in the case of hcp TMs (Figure 1).

Following the strategy used in previous studies on the bulk of TMs, ${ }^{19,20}$ three surface properties are investigated: One structural, here chosen as the interlayer distance relaxation, as e.g. obtained in metal single-crystal low-energy electron diffraction (LEED) experiments, including, when possible, inner layers relaxation. The main materials surface energetic 
property, the surface energy $\gamma$, is also evaluated, as defines the surface stability and, allegedly, the surface chemical activity. ${ }^{29}$ Note that surface energies are available from a compendium of $0 \mathrm{~K}$ extrapolated values obtained by a collection of measurements of TM surface tensions at/near melting temperatures. ${ }^{30}$ Finally, the surfaces work function, $\phi$, is here considered as a main electronic structure property, as inherently defined by the Fermi level $\left(\mathrm{E}_{\mathrm{F}}\right)$ position with respect the vacuum energy level, and often measured e.g. by scanning Kelvin probe force microscopy (SKPFM). ${ }^{31}$

Note that surface energies and work functions are two fundamental physicochemical parameters important to understand surface processes, including charge transfer, activity issues pivotal in surface catalyzed reactions, adsorptive processes, surface segregation and corrosion, growth rates, or the formation of grain boundaries, to name a few. These two properties are typically experimentally determined on mixed or polycrystalline samples exposing typically multiple facets, and those situations therefore should be better assessed considering the equilibrium shape of crystals. Consequently, here the different crystal orientations are weighted using the Wulff representation of crystal equilibrium shapes, ${ }^{32,33}$ although in the case of work functions, some single-crystal surface-specific values are available.

\section{Computational Details}

DFT based calculations have been performed using the Vienna ab initio simulation package (VASP) code, ${ }^{34}$ employing periodic boundary conditions, using the above commented VWN within LDA; PBE within GGA, and TPSS as an example of meta-GGA xc functionals. Furthermore, other GGA xc a priori adapted for bulk solids and surfaces are closely inspected, such as the PBE adapted for solids (PBEsol), ${ }^{35}$ or the revised PBE (RPBE) ${ }^{36}$ alleged to better describe the adsorption energies.

All TMs surfaces were modeled by 6-layered slabs using $(1 \times 1)$ surface unit cells with a minimum $10 \AA$ vacuum space, known to be enough to isolate slabs from their periodically repeated replicas, although in selected systems, such as in magnetic metals, a larger vacuum up to $30 \AA$ was used to avoid magnetic coupling. Slab surfaces were constructed from bulk optimized structures obtained using the very same xc functionals using the same computational setup. ${ }^{19,20}$ During structural optimization unit cells were kept fixed as in the bulk whereas all atomic positions were fully relaxed. 
The valence electron density was expanded in a plane-wave basis set with a $415 \mathrm{eV}$ cutoff for the kinetic energy, while the effect of the atomic cores into the valence electron density was described through the projector augmented wave (PAW) method. ${ }^{37}$ A tetrahedron smearing method of $0.2 \mathrm{eV}$ width was used to speed up the electronic convergence, ${ }^{38}$ yet final energy values were corrected to $0 \mathrm{~K}$. An electronic convergence criterion of $10^{-6} \mathrm{eV}$ was used, and ionic relaxation was considered converged when forces acting on atoms were smaller than $0.01 \mathrm{eV} / \AA$. The electronic structure calculations were by default non-spin polarized, except for magnetic Fe, Co, and Ni bulks and surfaces. An optimal MonkhorstPack $^{39}$ grid of $7 \times 7 \times 7$ k-points dimensions was found to be sufficient for accurate bulk total energy calculations in most stringent metals, and therefore used in all cases. ${ }^{19,20}$ In the case of slab calculations, a $7 \times 7 \times 1 \mathbf{k}$-points grid was used.

The surface energies, $\gamma$, are calculated following Eq. 1, where $E_{\text {slab }}$ is the energy of the optimized slab, $E_{\text {bulk }}$ the energy of a metal atom in its bulk environment, and $N$ the number of atoms in the employed slab model. The $A$ term is the exposed surface area exposed in each of the two exposed facets of the slab model.

$$
\gamma=\frac{E_{\text {slab }}-\left(N \cdot E_{\text {bulk }}\right)}{2 \cdot A}
$$

The work function, $\phi$, is defined as the minimum energy needed to remove an electron from a solid, located in its Fermi level $\left(\mathrm{E}_{\mathrm{F}}\right)$, and place it in the vacuum energy level, $V$, then;

$$
\phi=V-E_{F}
$$

where, in order to acquire $V$ the electron electrostatic potential energy is averaged for each surface along the normal to surface direction, until a constant value is found in the vacuum region, see Figure 2. $\mathrm{E}_{\mathrm{F}}$ level was obtained from the total density of states (DOS) sampled by circa 10,000 points, ensuring a numerical precision of $0.001 \mathrm{eV}$.

Finally, the surface relaxation is evaluated as the layer contraction/expansion percentage, $\Delta_{\mathrm{ij}}$, given with respect bulk environment. Note that for the studied TMs the interlayer spacing in between two vicinal layers $i$ and $j$ is constant for a given crystallographic direction in bulk, and so $\Delta_{\mathrm{ij}}$ is given as

$$
\Delta_{i j}=\frac{\delta_{i j}-\delta_{i j}^{b u l k}}{\delta_{i j}^{b u l k}} \cdot 100
$$

where $\delta_{i j}^{b u l k}$ is the bulk interlayer distance along the direction perpendicular to the surface whereas $\delta_{i j}$ is the equivalent interlayer distance in the slab model. In the bulk, the $i j$ index is 
a constant whereas in the slab refers to a pair of consecutive atomic layers. Hence, $i=1$ corresponds to surface layer, $j=2$ to the first subsurface layer. Thus $\delta_{12}$ would refer to the interlayer distance between the surface and the subsurface layers in the slab model. Within this definition, negative values of $\Delta_{\mathrm{ij}}$ imply an interlayer distance shortening, whereas positive values refer to interlayer distance lengthening.

\section{Results and Discussion}

For convenience, results concerning surface energies are presented first, followed by a discussion on the results for surface work functions. The analysis of the structural relaxation is then reported at the end of this section, followed by an overall discusison.

\section{Surface Energies}

In order to determine the best suited xc functional for this property we first compare the computed surface energies with the zero Kelvin extrapolated experimental data. Notice that such extrapolation is typically done from polycrystalline samples near melting temperatures, and likely correspond to an admixture of different exposed surfaces. ${ }^{30}$ However, it is unclear whether the zero Kelvin extrapolated data would belong to the most stable surface, or still to an admixture of competing surfaces. For that purpose we gained Wulff constructions such as those described in Figure 3. For each transition metal, the Wulff constructions were obtained from the calculated surface energies obtained for each $\mathrm{xc}$ functional. For $f c c$ and $b c c$ structures these were built either using the visualization for electronic and structural analysis (VESTA) package ${ }^{40}$ whereas the WinXMorph suite was used for the $h c p$ ones. ${ }^{41}$ There, for each TM and each xc, the constructed Wulff shapes provides the percentage of area exposed by each exposed surface. The specific computed surface energies, $\gamma^{\text {calc }}$ for each particular surface and each employed xc are found in Table S1 of the Supporting Information, alongside with the experimental surface energies, $\gamma^{\text {exp }}$. The obtained zero Kelvin Wulff contributions are listed in Table S2.

The performance of the different $\mathrm{xc}$ functionals in describing surface energies is analyzed in Figure 4 by comparing the computed estimates to experimental values. Notice that two comparisons are here made, either using those $\gamma^{\text {calc }}$ of most stable surfaces, consistently evaluated for each TM surface and xc, or Wulff shape averaged, thus considering contribution fractions of all surfaces of a given TM at a given xc level, $\gamma_{\text {Wulf }}^{\text {calc }}$. The latter provides a better comparison since, as mentioned, a large number of experimental values correspond to an average over exposed surfaces. For clarity, only linear fittings are plotted; 
although dispersion is evaluated by the linear regression factor, $R$, see Table $\mathrm{S} 3$ in Supporting Information, and the error analysis listed in Table 1. Note here that quantitative analysis of accuracy is made based on mean errors (ME), mean absolute errors (MAE), and mean absolute percentage errors (MAPE).

A close inspection on Figure 4 shows that the approximation of comparing calculated to measured surface energies assuming that experiments correspond to the most stable facets $-\gamma^{\text {calc }}$ case - can be justified since experimental trends are duly followed, especially when considering VWN and PBEsol functionals, which display linear regression with coefficients $R$ over 0.90 , slopes near unity, and intercepts of only -0.01 (VWN) and -0.12 (PBEsol) $\mathrm{J} / \mathrm{m}^{2}$. The performance of PBE and RPBE in this point can be considered as quite good, whereas metaGGA TPSS displays a poor adjustment, with an $R$ of only 0.51 , see Table S2.

Considering an admixture of surfaces, here simulated by Wulff averaged surface energy values $-\gamma_{\text {Wulff }}^{\text {calc }}$ case- one realizes that the a better fitting is obtained, see Figure 4. This is accompanied by slightly better $R$ coefficients, slopes, and intercepts, see Table S2. The only exception to this fact is RPBE; The Wulff averaged calculated value leads to a slope increase from 0.85 up to 0.95 , and the intercept changes from $-0.34 \mathrm{~J} / \mathrm{m}^{2}$ to $0.18 \mathrm{~J} / \mathrm{m}^{2}$. This apparent better fit is indeed not, as $R$ drops from 0.88 to 0.56 . As a matter of fact, the poor description of surface energies at RPBE does not only apply to the most stable surfaces, but also to others, which scales to the admixture of all contemplated surfaces, see Table S1. This unrealistic treatment of surfaces on an equal foot upraises the Wulff overall surface energies in a quite unbalanced fashion, leading to a higher dispersion. Therefore, the apparently better fitting is an artifact stemming from the poor performance of RPBE in describing surface energies, rather than a proper description based on a realistic physical description.

A quantitative analysis based on the statistical errors is shown in Table S1. Notice how ME and MAE values for $\gamma^{\text {calc }}$ differ, meaning that the different xc functionals do not exhibit an absolute systematic error, i.e. having constant over- and underestimations. Nevertheless, a general underestimation is noticeable and actually surface energies tend to follow the bonding strength. The gradation of bonding strength on TMs bulks has been established previously from the cohesive energy, $\mathrm{E}_{\mathrm{coh}}$, being $\mathrm{VWN}>\mathrm{PBEsol}>\mathrm{PBE}>$ RPBE. ${ }^{19}$ According to the bond-cutting model, surface energies are directly proportional to $\mathrm{E}_{\mathrm{coh}}{ }^{42}$ and, therefore, surface energies follow the very same trend, yet being always underestimated. As shown in Table S4, a crystallographic decomposed quantitative analysis 
yields differences among structures, which can be quite mild for xc functionals such as VWN, with MAPE variations below 6\% using $\gamma^{\text {calc }}$, and quite severe for others, such as TPSS, with variations of MAPE above $30 \%$. When considering $\gamma_{\text {Wulff }}^{\text {calc }}$, the differences became more acute, such as below 9\% (VWN) and above 49\% (TPSS). Clearly, the trends are not uniform, and the only particularly crystallographic better description deviation is only noted for $b c c$ TMs, where PBEsol seems to be better suited than VWN, with MAPEs slightly above $15 \%$, and a clearly worse description by TPSS - MAPEs above 57\% - and a fair improvement of RPBE, with MAPEs around 20\%.

In any case the average performance is acceptable with VWN being the best suited xc in describing surface energies, with a deviation of circa $12 \%$. Present results excellently agree within $4 \%$ to previous results on a subset of $f c c(111)$ surfaces as obtained at LDA and GGA levels. ${ }^{19}$ Concerning comparison with experiments, notice on one hand that the average precision of the surface tension experiments is $\pm 2 \%$, a point that partially amends the disagreement of the present estimates. Further than that, notice that in the present study we neglected the contribution to the surface energy of other defects, including nanoparticles edges and corners, facet terraces and kinks, as well as other higher order Miller or MillerBravais indices facets, which, if accounted for, could overall slightly increase the agreement between calculated and experimental surface energy values.

\section{Work functions}

Next, we discuss the performance of the different functionals on predicting surface work functions. The complete set of calculated values is reported in Table S5 in the Supporting Information. Following the same procedure as in the previous section, we rely on the Wulff constructions to obtain an averaged value of $\phi$ to be compared with the experimental values as obtained from polycrystalline samples. ${ }^{31}$ However, for this observable, there is also a significant number of surface specific measurements obtained from TM single crystals cut in the desired studied directions. Therefore, for this subset of data, a direct comparison is possible and the list of experimental values is reported in Table S5 of the Supporting Information. Notice that 26 polycrystalline values are accessible — all TMs except synthetic Tc. Concerning well defined surfaces, 50 experimental values are available, which correspond to $\sim 62 \%$ of the studied cases. Concerning temperature effects, we here compare $0 \mathrm{~K}$ estimated of $\phi$ to experimental values normally obtained at or near room temperature. However, there is experimental evidences that the transition metal work functions change with temperature by between $10^{-4}$ to $10^{-6} \mathrm{eV} / \mathrm{K} .^{43,44}$ Therefore, the 
disagreement due to thermal effects at standard conditions cannot exceed $0.04 \mathrm{eV}$. Consequently, this small deviation is neglected in the oncoming analysis and discussion.

Two sets of values are used for comparison to polycrystalline values, $\phi_{W u l f}^{\text {calc }}$, using Wulff-averaged values, or single crystal data well defined surfaces, $\phi_{\text {Single }}^{\text {calc }}$. The trends from linear fittings are reported in Figure 5, whereas the corresponding statistical analysis is summarized in Table 2. As one can see, a better correlation is obtained when using the oneto-one comparison to single crystal surfaces, as expected. Note that, for this property, and at variance with surface energies, VWN does not show a good fitting, and actually the calculated set that seems to better meet the experimental trend is PBE for both single crystal and polycrystalline samples. Slight quantitative differences are found depending on the utilization of single crystal or polycrystalline values, see Table 2, although the performance decreases as $\mathrm{PBE}>$ PBEsol $>$ RPBE $>$ TPSS $>$ VWN for the single-crystal analysis. This trend is almost maintained when comparing to the polycrystalline data, where only PBEsol and RPBE swap positions. Notice in Figure 5, that, at variance to trends for calculated surface energies, the trends on workfunctions appear to show a somewhat overestimation of large $\phi$ values, and an underestimation of small $\phi$ ones, with transient points located at $\sim 4$ and $\sim 3 \mathrm{eV}$ for single-crystal and polycrystalline cases, respectively. For the oncoming analysis, we decided to better rely on the single-crystal data, given that slab models provide a realistic representation of these systems. Thus, we neglect other higher order Miller index surfaces, and other low-coordinated sites on the nanoparticle approximation, which can affect the simulated values. From this analysis, PBE appears as the best performing xc functional.

Considering the linear fittings, some caveats ought to be commented. The slope $(a)$, intercept $(b)$, and $R$ factors of Figure 5 linear fittings are reported on Table S6. These generally show: $i$ ) a clear deviation with respect the expected linear trend for the $\phi_{W u l f f}^{\text {calc }}$ case, with slopes overestimated well above 1.5, and intercepts far from zero by a few eV. Moreover, the data dispersion is reflected by exceedingly small $R$ regression coefficients, below 0.8 . When considering $\phi_{\text {Single }}^{\text {calc }}$ data, the slopes reduce to 1.45 or below, and intercepts significantly approaching to the ideal value of zero by $0.8-1.5 \mathrm{eV}$. The TPSS $\phi_{\text {Single }}^{\text {calc }}$ case is to be highlighted as displays $a$ and $b$ of 1.05 and $0.46 \mathrm{eV}$, respectively, although this improvement in the linear fitting is gained at the expense of a poorer dispersion of data, with a reduced regression coefficient, a common denominator in the fitting to single-crystal data. This said, a further close inspection to the crystallographic itemized statistics, shown in Table 
$\mathrm{S} 7$, indicates a general pattern in the sense that work function of $f c c$ metal surfaces are better described than those of $h c p$ and $b c c$ ones, and for this situation, TPSS performs better. However, $b c c$ are better described when comparing to polycrystalline data, although there the best description comes from the PBE functional. At variance with the above commented case of surface energies, the work function experimental accuracy, below $\pm 0.3 \%$, barely helps in concealing estimates and measurements.

\section{Interlayer distance}

Finally, as far as surface relaxation is concerned, a limited amount of experimental data is available, most focused on the surface and first subsurface interlayer distance, $\delta_{12}$. In addition, the experimental reference data for the full set of surfaces and TMs is not complete with a sensible heterogeneity of values with different accuracies and experimental precision, sometimes contradictory for a particular TM surface. Because of this we selected as reference data those which display $i$ ) higher precision, $i$ ) a larger amount of interlayer distances, and iii) for $i$ ) and ii) being on equal foot, those obtained most recently. The full set of data and concomitant references are reported in Table S8 of the Supplementary Information, as well as those used values highlighted in bold.

The calculated data at all the explored xc levels, including the three different interlayer relaxation values $\left(\Delta_{12}, \Delta_{23}\right.$, and $\left.\Delta_{34}\right)$ are contained in Table S9 of the Supplementary Information. However, the relaxation percentage as usually provided in the experiments is of little use in evaluating xc performance, as it is already a referenced data. Because of this, it seems more justified to directly compare the xc computed interlayer distance, $\delta_{i j}$, to the experimental values, derived from the experimentally available interlayer relaxations. To obtain $\delta_{i j}^{\text {exp }}$, the experimental interlayer percentage relaxations were applied to the $0 \mathrm{~K}$ extrapolated interlayer distances. ${ }^{20}$ The computed values are reported in Table S10 of the Supplementary Information. The performance of the different methods on predicting $\delta_{i j}$ has been analyzed statistically and results are reported in Table 3. Graphically, the calculated values nicely fit the experimental set of values, as seen in Figure 6, with very small deviations of typically around $0.15-0.22 \AA$ (Table 3 ) with little variations in between the different considered xc functionals. This is also reflected in the data from the linear fitting shown in Table S11 in the Supplementary Information, with slopes near $\sim 0.8$, intercepts below $0.4 \AA$, and $\mathrm{R}^{2}$ circa 0.8 . This is in agreement with the rather good description of geometrical structure by LDA, GGA, and meta-GGA xc functionals in general, and on bulk TMs in particular. ${ }^{19,20}$ Despite of this, Figure 6 shows that PBE performs slightly better with 
comparison to experiment superior to that corresponding to other explored xc functionals. This is aligned with the PBE smallest MAPE value in Table 3. However, this result varies with the crystallographic structure, shown in Table S12, with bcc being the most accurate for this property. In addition, it is important to remark that experiments show a wide range of precision, $\pm 1.5 \%$ on average, which actually closes the gap of any DFT calculation, and actually, the differences among functionals seem to be not so acute as the previously found for $\gamma$ and $\phi$ observables.

\section{Overall Functional Assessment}

To summarize, a general view of the performance, in terms of MAPE, of the different explored functionals on predicting surface relaxations, surface energy, and surface work function, is provided. Notice that concerning the assessment of the different functionals, results evidence heterogeneity on the properties under study - even crystallographic groups, and specific TMs, see Figure S1. Even so, an overall better performance for specific cases is observed for VWN concerning $\gamma$, and for RPBE on $\phi$, yet for the latter property the mean best performance is again for PBE. On average, VWN is best adapted to surface energies, and PBE to work functions and interlayer distances. However, for a general assessment of surface properties, one can add up the obtained MAPEs for each xc functional under study, as shown in Figure 7. A close inspection reveals that, according to this criterion, the most balanced surface properties are provided by PBEsol, despite not being the functional providing better fitting between calculations and experiment for the properties under inspection. Further than that, combining the present analysis with that for TMs bulk properties - cohesive energies, $E_{c o h}$, bulk moduli, $B_{0}$, and shortest interatomic distances $\delta$ - as obtained earlier with the same procedure here applied, ${ }^{19,20}$ the most accurate xc functional is PBE, yet closely followed by PBEsol and TPSS.

In this sense one would not advice VWN and RPBE when computing TMs bulk or surfaces properties, although VWN is particularly suited when estimating surface energies. All that said, RPBE has been claimed to represent an improvement regarding the adsorption of main group molecules on TM surfaces, ${ }^{36}$ a point of the scope of the present study, although the metaGGA TPSS functional also represents an improvement in the description of the thermochemistry of main group systems, a point that, coupled to present good performance, can place TPSS in a best-compromise situation when studying the interaction of atoms and/or molecules on TM surfaces. ${ }^{20,24}$ Moreover, the present study further reveals that, at least for TM extended systems, the development of new xc functionals needs to consider a broad 
number of properties much as in the same spirit of the G2 and related datasets used in molecular quantum chemistry. ${ }^{45}$ This has been exemplified here by assessing the performance of a series of functional on predicting surface (and bulk) properties, which hopefully serves as a spur and guidance for future DFT xc improvements.

Along this line, further functional research targeting transition metal properties description should address the possible effect of dispersive forces in any of the explored metals, properties, and functionals, a point here so far not contemplated. A recent study by Patra et al. showed that the Strongly Constrained Appropriately Normed (SCAN) semilocal metaGGA functional ${ }^{46}$ —including intermediate-range van der Waals (vdW) descriptionand the addition of Vydrov-Voorhis vdW to SCAN (SCAN+rVV10) ${ }^{47}$ —including as well long-range vdW interactions - affected the surface energies and work functions or a subset of seven $f c c$ TMs, increasing the surface energies by $\sim 10 \%$, and work functions by $\sim 3 \%$, but, more importantly, SCAN-rVV10 showed the best description regardless of the property, ${ }^{48}$ although such a statement remains to be confirmed by considering a broader set of TMs as this is the case in the present work.

\section{Conclusions}

A survey on the performance of various widely-used DFT xc functionals in describing surface observables of all transition metals with $f c c, b c c$, or $h c p$ crystallographic structures has been presented. The properties under scrutiny include structural (interlayer distances), energetic (surface energies), and electron transfer (work functions). These properties were estimated on appropriate models of low Miller index (Miller-Bravais for $h c p$ ) surfaces and obtained with broadly used xc functionals within the three first rungs of Jacob's ladder, including VWN LDA, PBE, PBEsol, and RPBE within GGA, and TPSS as a metaGGA representative. A comparison to a large set of available experimental data, including singlecrystal when available, is provided. Comparison to polycrystalline values is also carried out with the help of Wulff construction for equilibrium shape of nanoparticles. This strategy allowed to estimate surface properties averaged according to the proportion of exhibited facets in polycrystalline samples.

Results show that no $\mathrm{xc}$ is best at simultaneously describing all surface properties with slight variations depending on the property, transition metal, even on the crystallographic arrangement. Despite of this, present results show that, on average terms, 
VWN is best suited in predicting surface energies, whereas PBE provides the best description for interlayer atomic distances and also for work functions. However, PBEsol xc is advised for an mean, balanced description of surface properties, yet when simultaneously considering both bulk and surface properties, PBE is recommended. The present results highlight the importance of fitting xc functionals to a diverse range of properties and transition metal extended systems for an improved performance.

\section{Acknowledgements}

This work was supported by Spanish MINECO/FEDER grant CTQ2015-64618-R and, in part, by Generalitat de Catalunya grants (2014SGR97 and XRQTC), and the NOMAD Center of Excellence project; the latter project has received funding from the European Union Horizon 2020 research and innovation programme under grant agreement No 676580. F.V. thanks the Spanish MINECO for postdoctoral contracts under the Ramón y Cajal (RYC-201210129) program, and F.I. acknowledges additional support through the ICREA Academia award for excellence in research. 
Figure 1. Schematic representation of the studied (001), (011), and (111) surfaces for $f c c$ and

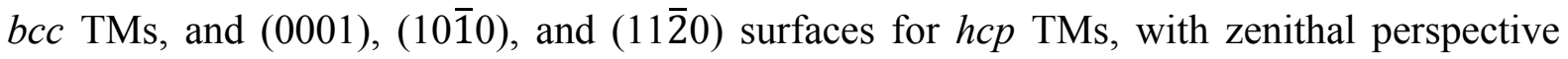
views. The employed $(1 \times 1)$ unit surface cells are shown in solid black lines. Atomic positions are denoted by colored spheres, which turn darker when going subsurface. The particular cases presented are $\mathrm{Nb}(b c c), \mathrm{Pt}(f c c)$, and $\mathrm{Ru}(h c p)$, all optimized PBE.
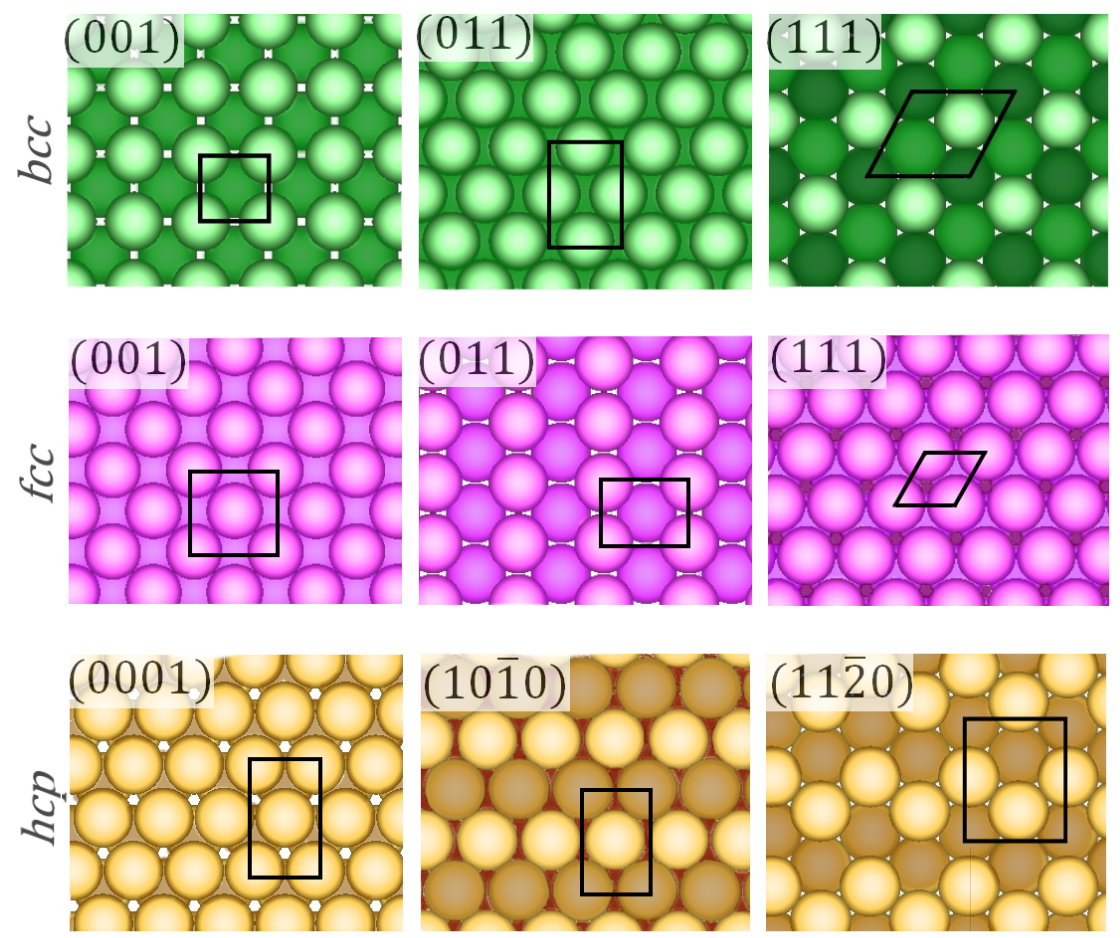
Figure 2. Electrostatic potential energy for a prototypal 6-layered slab - Mo(001) surface TPSS - as a function of vacuum axis length.

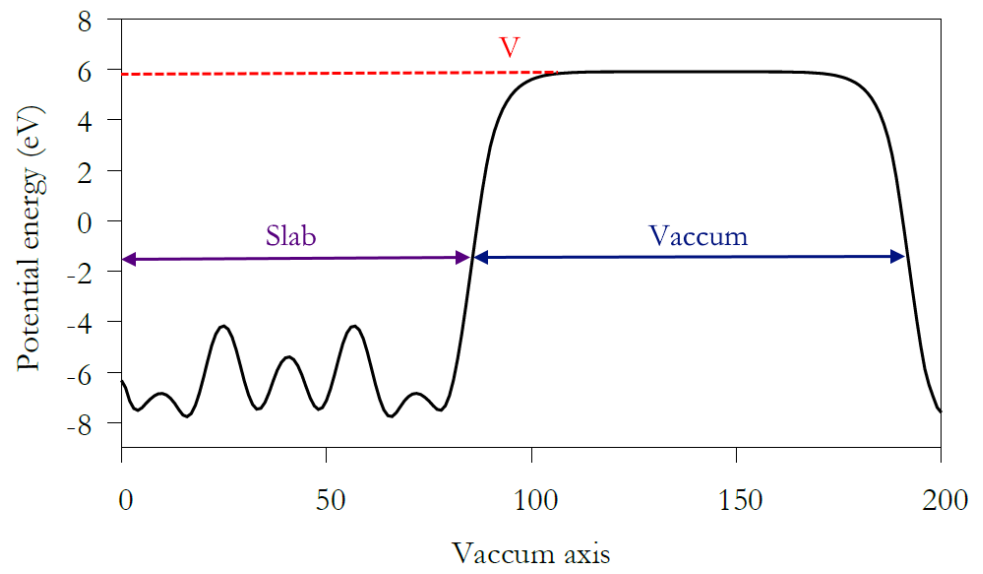


Figure 3. Exemplary Wulff shape representations of different TMs as predicted from the different $\mathrm{xc}$ functionals. The green shape belongs to $\mathrm{W} b c c$ obtained with VWN xc; the pink shape to Ni $f c c$ obtained with PBEsol, and the yellow one to $\mathrm{Zr} h c p$ obtained with TPSS. Tags for the different surfaces are located nearby the exposed facets.

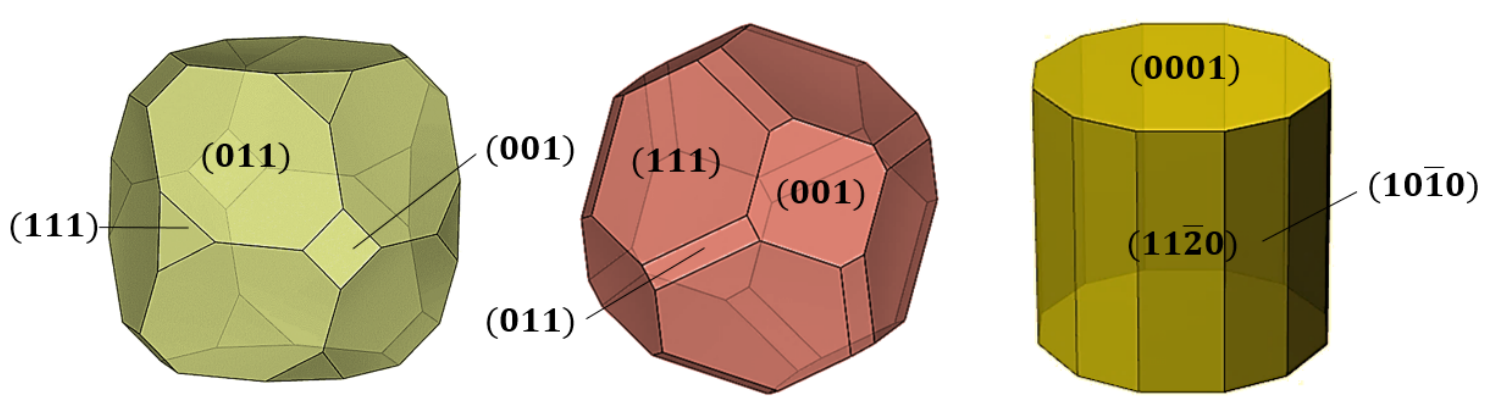


Figure 4. Comparison of the linear fitting between calculated (calc) and experimental (exp) surface energies for the different explored xc functionals. The top panel considers only the most stable surface, $\gamma^{\text {calc }}$, whereas in the bottom panel Wulff shape averaged values, $\gamma_{\text {Wullf }}^{\text {calc }}$, are compared.

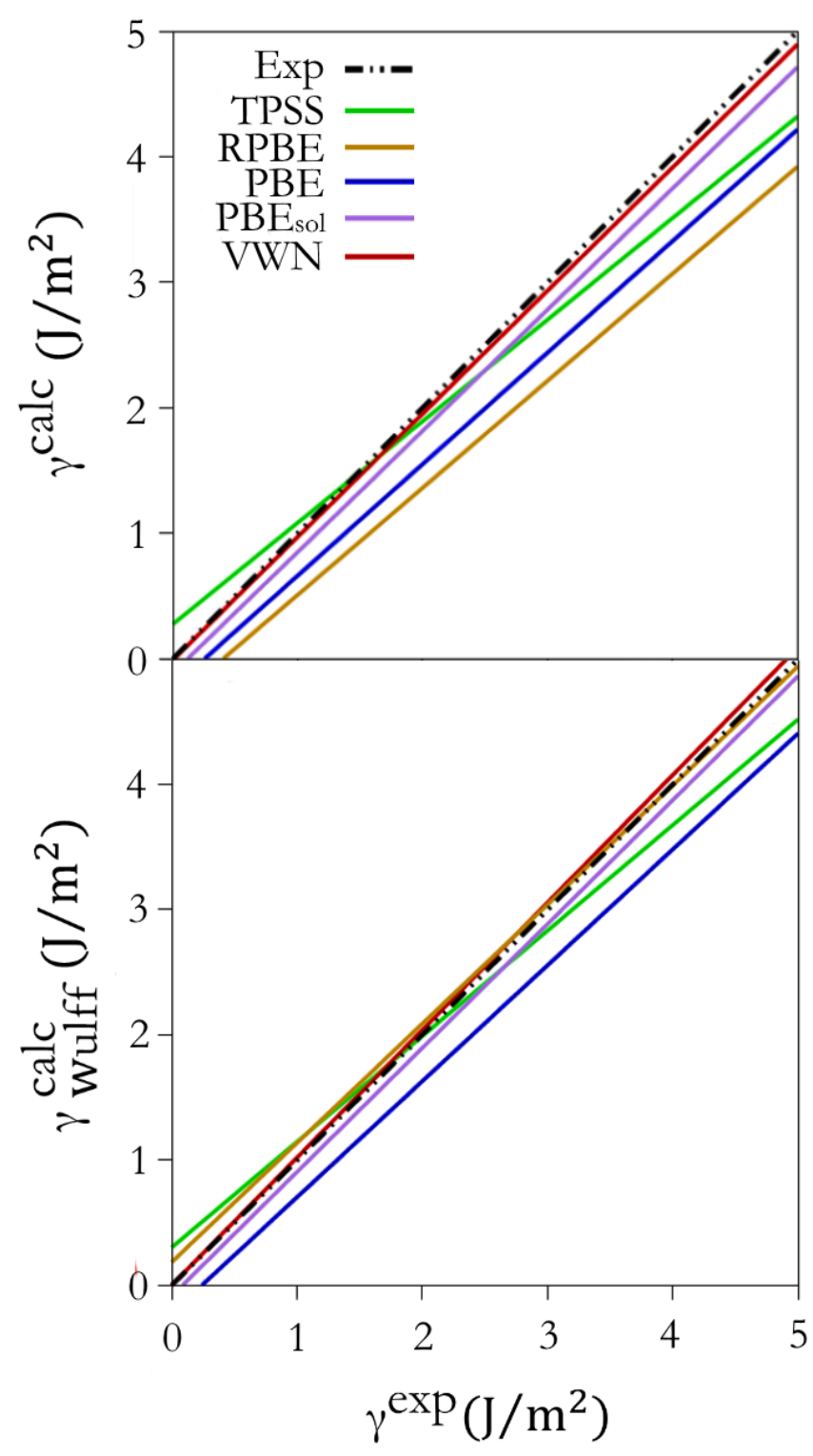


Figure 5. Comparison of the linear fitting between calculated (calc) and experimental (exp) work functions for the different explored xc functionals. The top panel considers only single crystal data, $\phi_{\text {Single }}^{\text {calc }}$, whereas in the bottom panel Wulff shape averaged values, $\phi_{\text {Wulff }}^{\text {calc }}$, are compared. All values are given in $\mathrm{eV}$.

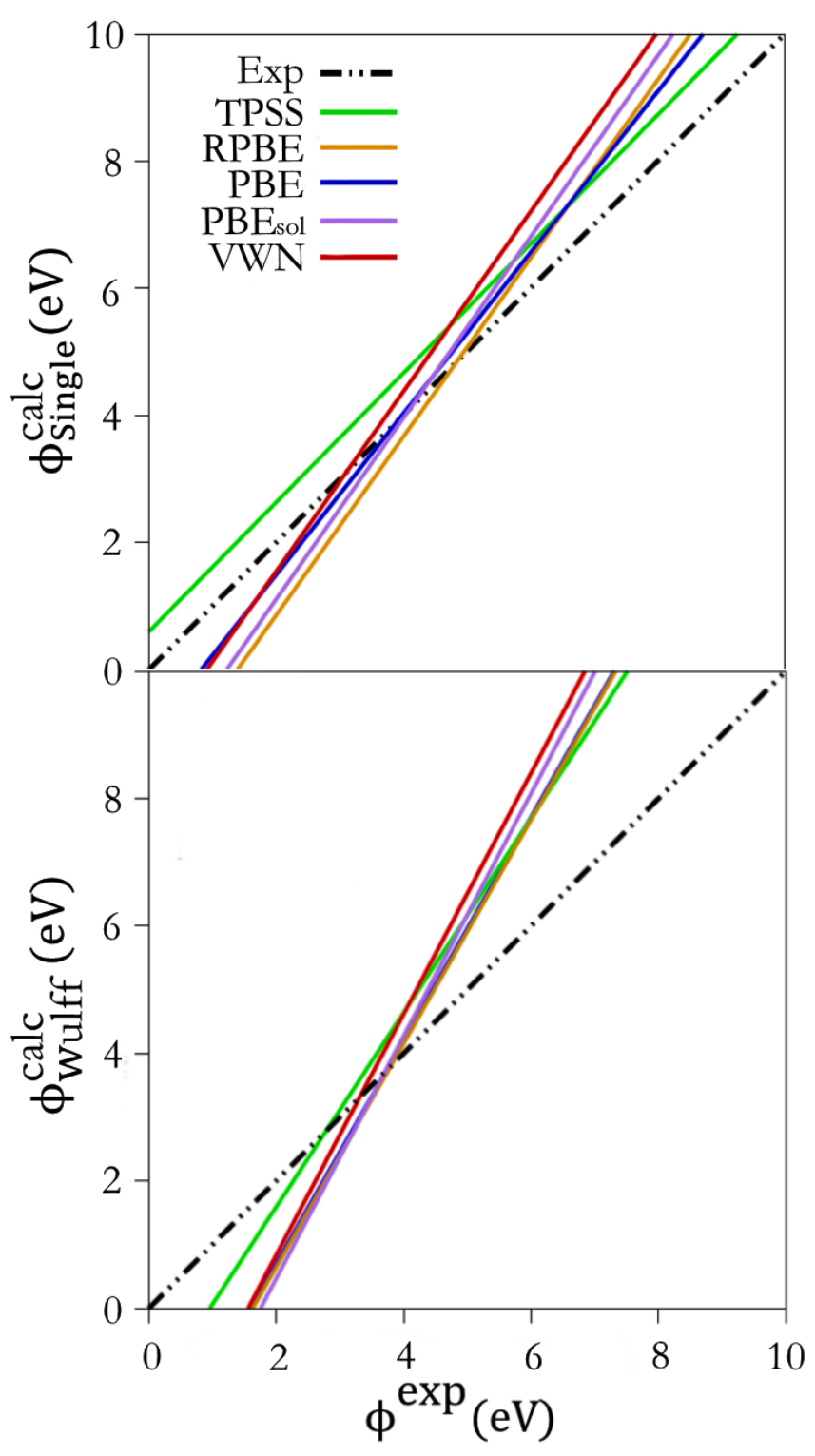


Figure 6. Comparison of $\delta_{i j}$ MAPE to experimental data, $\delta_{i j}^{\text {exp }}$, as calculated and adjusted to linear regression for each functional.

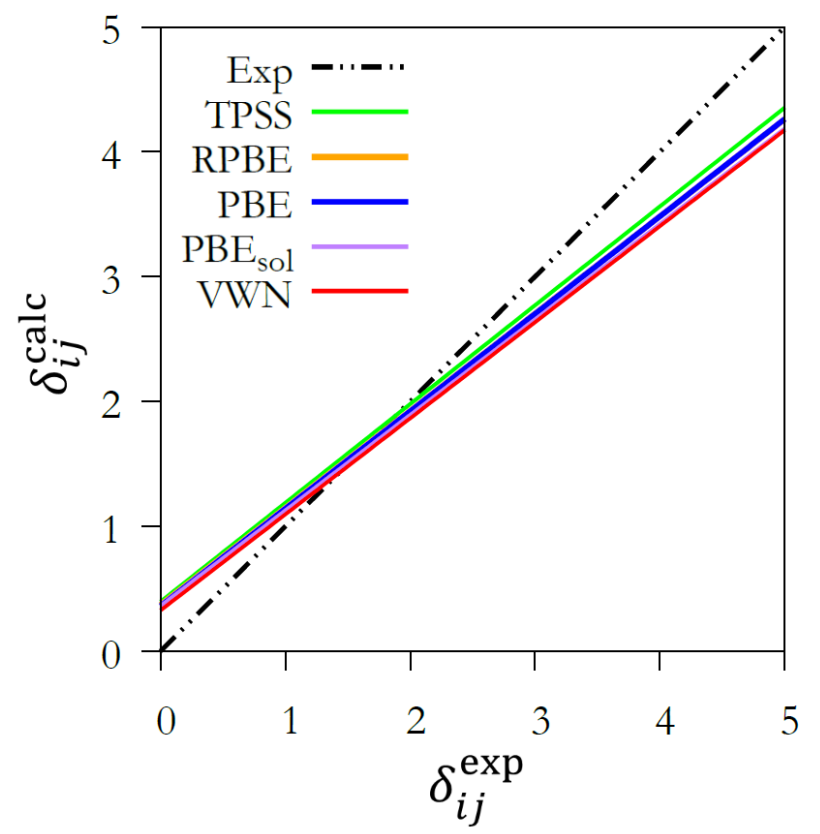


Figure 7. Summary of xc functional accuracy in describing bulk and surface related properties, according to the added MAPE values. Accuracies of distance related properties have been arbitrarily enlarged 5 times to help in clearly differentiating the performance of the different $x c$ functionals on them.

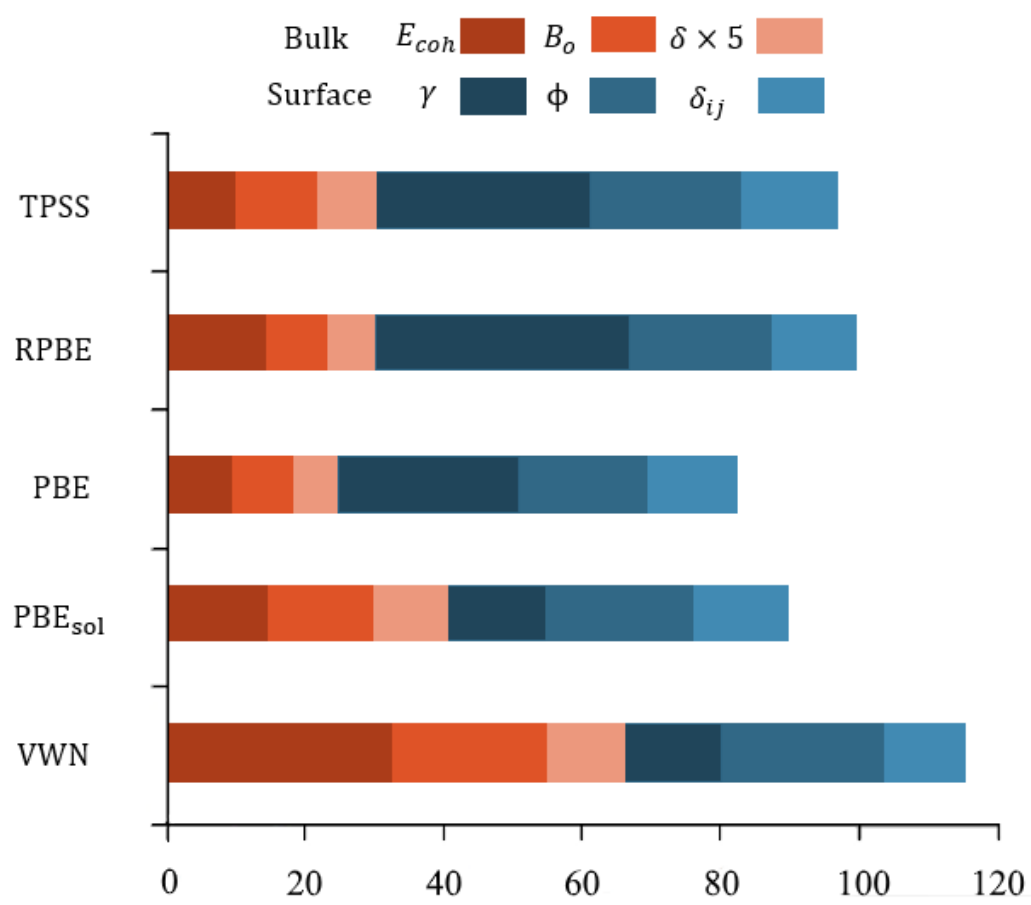


Table 1. Mean error (ME), mean absolute error (MAE), and mean absolute percentage error (MAPE) of calculated most stable surface energies $\gamma^{\text {calc }}$ with respect experiments, as well as Wulff construction weighted values, $\gamma_{\text {Wullf }}^{\text {calc }}$, as obtained at VWN, PBEsol, PBE, RPBE, and TPSS xc levels. All values are given in $\mathrm{J} / \mathrm{m}^{2}$, except for MAPE, given in $\%$.

\begin{tabular}{lccccc}
\hline \multicolumn{5}{c}{$\gamma^{\text {calc }}\left(\mathbf{J} / \mathbf{m}^{2}\right)$} \\
VWN & PBEsol & PBE & RPBE & TPSS \\
\hline \hline ME & -0.05 & -0.20 & -0.49 & -0.69 & -0.17 \\
MAE & 0.28 & 0.34 & 0.57 & 0.73 & 0.72 \\
MAPE & 13.48 & 16.58 & 27.11 & 34.84 & 31.42 \\
\multicolumn{5}{c}{$\gamma_{\text {Wullf }}^{\text {calc }}\left(\mathbf{J} / \mathbf{m}^{2}\right)$} \\
ME & 0.04 & -0.11 & -0.40 & 0.07 & -0.06 \\
MAE & 0.27 & 0.29 & 0.49 & 0.96 & 0.68 \\
MAPE & 12.24 & 14.02 & 23.25 & 48.49 & 29.04 \\
\hline
\end{tabular}


Table 2. Statistical analysis based on ME, MAE, and MAPE of calculated work functions with respect experiments (single crystal surfaces, $\phi_{\text {Single }}^{\text {calc }}$, and polycrystalline values, $\phi_{W u l f f}^{\text {calc }}$ ) as obtained at VWN, PBEsol, PBE, RPBE, and TPSS levels. All values are given in eV, except for MAPE, given in $\%$.

\begin{tabular}{lccccc}
\hline & \multicolumn{5}{c}{$\boldsymbol{\phi}_{\text {Single }}^{\text {calc }}(\mathrm{eV})$} \\
& VWN & PBEsol & PBE & RPBE & TPSS \\
\hline \hline ME & 0.79 & 0.38 & 0.30 & 0.07 & 0.69 \\
MAE & 1.13 & 1.06 & 1.02 & 1.08 & 1.10 \\
MAPE & 22.85 & 21.42 & 20.77 & 22.04 & 22.41 \\
& \multicolumn{5}{c}{$\boldsymbol{\phi}_{\text {Wulff }}^{\text {calc }}(\mathbf{e V})$} \\
ME & 1.10 & 0.77 & 0.61 & 0.57 & 0.97 \\
MAE & 1.25 & 0.99 & 0.91 & 0.94 & 1.18 \\
MAPE & 26.49 & 20.91 & 19.39 & 20.15 & 25.36 \\
\hline
\end{tabular}


Table 3. Statistical analysis based on ME, MAE, and MAPE of calculated interlayer distances, $\delta_{i j}$, compared to experimental ones, $\delta_{i j}^{e x p}$, as obtained at VWN, PBEsol, PBE, RPBE, and TPSS levels. All values are given in pm, except for MAPE, given in $\%$

\begin{tabular}{lccccc}
\hline & \multicolumn{5}{c}{$\boldsymbol{\delta}_{\boldsymbol{i} \boldsymbol{j}}^{\text {calc }}(\AA)$} \\
& VWN & PBEsol & PBE & RPBE & TPSS \\
\hline \hline ME & -0.08 & -0.07 & -0.03 & -0.01 & 0.03 \\
MAE & 0.18 & 0.17 & 0.15 & 0.16 & 0.22 \\
MAPE & 11.51 & 10.87 & 10.11 & 10.36 & 13.76 \\
\hline
\end{tabular}


Table of Contents

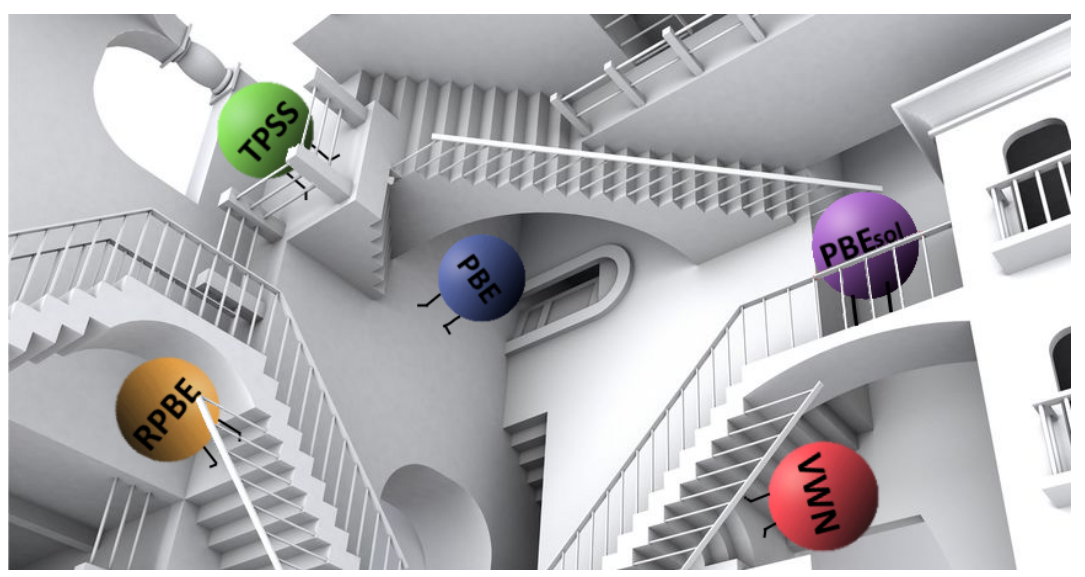




\section{ASSOCIATED CONTENT}

\section{Supporting Information}

The Supporting Information is available free of charge on the ACS Publications website at DOI:

Table S1 contains the computed surface energies for each TM, functional, and surface, as well as the experimental values. Table S2 encompasses the percentage of expression of each surface for each TM as self-consistently obtained at each DFT level using the Wulff constructions. Tables S3, S6, and S11 contain the linear adjustment parameters corresponding to Figures 4, 5, and 6, respectively. Tables S4, S7, and S12 provide the statistical analysis of Tables 1-3 but decomposed according to $f c c, b c c$, and $h c p$ crystallographic structure families. Table S5 contains the computed workfunctions for each TM, functional, and surface, as well as experimental polycrystalline and single-crystal data. Table S8 encompasses the experimental interlayer distances found in the literature, highlighting those used as reference data. Table S9 and S10 provide the computed interlayer relaxations and distances. Figure S1 shows, color-coded, the best functional for each bulk and surface TM property.

\section{References}

1. Parr, R.G.; Yang, W., Density Functional Theory of Atoms and Molecules. Oxford University Press: 1989.

2. Perdew, J. P.; Schmidt, K., Jacob's Ladder of Density Functional Approximations for the Exchange-Correlation Energy. AIP Conf. Proc. 2001, 577, 1.

3. Medvedev, M.G.; Bushmarinov, I.S.; Sun, J.; Perdew, J.P.; Lyssenko, K.A., Density Functional Theory is Straying from the Path Toward the Exact Functional. Science 2017, $355,49-52$.

4. Sousa, S. F.; Fernandes, P. A.; Ramos, M. J., General Performance of Density Functionals. J. Phys. Chem. A 2007, 111, 10439-10452.

5. Peverati, R.; Truhlar, D. G., An Improved and Broadly Accurate Local Approximation to the Exchange-Correlation Density Functional: The MN12-L Functional for Electronic Structure Calculations in Chemistry and Physics. Phys. Chem. Chem. Phys. 2012, 14, 13171-13174. 
6. Tomić, S.; Montanari, B.; Harrison, N. M., The Group III-V's Semiconductor Energy Gaps Predicted Using the B3LYP Hybrid Functional. Phys. E 2008, 40, 2125-2127.

7. Viñes, F.; Lamiel-Garcia, O.; Ko, K. C.; Lee, J. Y.; Illas, F., Systematic Study of the Effect of HSE Functional Internal Parameters on the Electronic Structure and Band Gap of a Representative Set of Metal Oxides. J. Comput. Chem. 2017, 38, 781-789.

8. Peverati, R.; Truhlar, D. G., Performance of the M11-L Density Functional for Bandgaps and Lattice Constants of Unary and Binary Semiconductors. J. Chem. Phys. 2012, 136, 134704.

9. Viñes, F.; Lamiel-Garcia, O.; Bromley, S. T.; Illas, F. Size Dependent Structural and Polymorphic Transitions in ZnO: from Nanocluster to Bulk. Nanoscale 2017, 9, 1006710074.

10. Lamiel-Garcia, O.; Ko, K. C.; Lee, J. Y.; Bromley, S. T.; Illas, F., When Anatase Nanoparticles Become Bulklike: Properties of Realistic $\mathrm{TiO}_{2}$ Nanoparticles in the 1-6 nm Size Range from All Electron Relativistic Density Functional Theory Based Calculations. J. Chem. Theory Comput. 2017, 13, 1785-1793.

11. Viñes, F.; Gomes, J. R. B.; Illas, F., Understanding the Reactivity of Metallic Nanoparticles: Beyond the Extended Surface Model for Catalysis. Chem. Soc. Rev. 2014, 43, 4922-4939.

12. Yang, M. H.; Yang, Y. H.; Liu, Y. L.; Shen, G. L.; Yu, R. Q., Platinum NanoparticlesDoped Sol-Gel/Carbon Nanotubes Composite Electrochemical Sensors and Biosensors. Biosens. Bioelectron. 2006, 21, 1125-1131.

13. Frenking, G., Theoretical Aspects of Transition Metal Catalysis. Springer Berlin Heidelberg: 2005.

14. Thomas, J. M.; Thomas, W. J., Principles and Practice of Heterogeneous Catalysis. 2 ed.; Wiley-VCH: Weinheim, 2015.

15. Anderson, J. A.; Fernández-García, M., Supported Metals in Catalysis. Imperial College Press: London, 2011.

16. Wu, J.; Yang, H., Platinum-Based Oxygen Reduction Electrocatalysts. Acc. Chem. Res. 2013, 46, 1848-1857.

17. Van Santen, R.A.; Neurock, M., Molecular Heterogeneous Catalysis. Wiley-VCH: 2006.

18. Perdew, J. P.; Ernzerhof, M.; Burke, K., Rationale for Mixing Exact Exchange with Density Functional Approximations. J. Chem. Phys. 1996, 105, 9982. 
19. Janthon, P.; Kozlov, S. M.; Viñes, F.; Limtrakul, J.; Illas, F., Establishing the Accuracy of Broadly Used Density Functionals in Describing Bulk Properties of Transition Metals. J. Chem. Theory Comput. 2013, 9, 1631-1640.

20. Janthon, P.; Luo, S.; Kozlov, S. M.; Viñes, F.; Limtrakul, J.; Truhlar, D. G.; Illas, F., Bulk Properties of Transition Metals: A Challenge for the Design of Universal Density Functionals. J. Chem. Theory Comput. 2014, 10, 3832-3839.

21. Notario-Estévez, A.; Kozlov, S. M.; Viñes, F.; Illas, F., Electronic-structure-based material descriptors: (in)dependence on self-interaction and Hartree-Fock exchange. Chem. Commun. 2015, 51, 5602-5605.

22. Determan, J. J.; Poole, K.; Scalmani, G.; Frisch, M. J.; Janesko, B. G.; Wilson, A. K., Comparative Study of Nonhybrid Density Functional Approximations for the Prediction of $3 d$ Transition Metal Thermochemistry. J. Chem. Theory Comput. 2017, 13, 49074913.

23. Perdew, J. P.; Burke, K.; Ernzerhof, M., Generalized Gradient Approximation Made Simple. Phys. Rev. Lett. 1996, 77, 3865-3868.

24. Tao, J.; Perdew, J. P.; Statoverov, V. N.; Scuseria, G. E., Climbing the Density Functional Ladder: Nonempirical Meta-Generalized Gradient Approximation Designed for Molecules and Solids. Phys. Rev. Lett. 2003, 91, 146401.

25. Fajín, J. L. Viñes, F. Cordeiro, N. D. S.; Illas, F.; Gomes, J. R. B. Effect of the Exchange-Correlation Potential on the Transferability of Brønsted-Evans-Polanyi Relationships in Heterogeneous Catalysis. J. Chem. Theory and Comput. 2016, 12, 2121 2126

26. Sun, J.; Xiao, B.; Fang, Y.; Haunschild, R.; Hao, P.; Ruzsinszky, A.; Csonka, G. I.; Scuseria, G. E.; Perdew, J. P. Density Functionals that Recognize Covalent, Metallic, and Weak Bonds. Phys. Rev. Lett. 2013, 111, 106401.

27. Sun, J.; Remsing, R. C.; Zhang, Y.; Sun, Z.; Ruzsinszky, A.; Peng, H.; Yang, Z.; Paul, A.; Waghmare, U.; Wu, X.; Klein, M. L.; Perdew. J. P. Accurate First-Principles Structures and Energies of Diversely Bonded Systems from an Efficient Density Functional. Nat. Chem. 2016, 8, 831-836.

28. Vosko, S. H.; Wilk, L.; Nusair, M., Accurate Spin-Dependent Electron Liquid Correlation Energies for Local Spin Density Calculations: A Critical Analysis. Can. J. Phys. 1980, 58, 1200. 
29. Zhuang, H.; Tkalych, A.J.; Carter, E., Surface Energy as a Descriptor of Catalytic Activity. J. Phys. Chem. C 2016, 120, 23698.

30. Mills, K. C.; Su, Y. C., Review of Surface Tension Data for Metallic Elements and Alloys: Part 1 - Pure Metals. Int. Mater. Rev. 2006, 51, 329.

31. Helbert B. M., The Work Function of the Elements and its Periodicity. J. Appl. Phys. $1977,48,4729$.

32. Wulff, G., Zur Frage der Geschwindigkeit des Wachstums und der Auflösung von Krystallflächen. Z. Kristallogr. Mineral. 1901, 34, 449.

33. Viñes, F.; Gomes, J. R. B.; Illas, F., Understanding the Reactivity of Metallic Nanoparticles: Beyond the Extended Surface Model for Catalysis. Chem. Soc. Rev. 2014, $43,4922$.

34. Kresse, G.; Furthmüller, J., Efficiency of Ab-Initio Total Energy Calculations for Metals and Semiconductors Using a Plane-Wave Basis Set. J. Comput. Mater. Sci. 1996, 6, 15.

35. Perdew, J. P.; Ruzsinszky, A.; Csonka, G. I.; Vydrov, O. A.; Scuseria, G. E.; Constantin, L. A.; Zhou, X.; Burke, K., Restoring the Density-Gradient Expansion for Exchange in Solids and Surfaces. Phys. Rev. Lett. 2008, 100, 136406.

36. Hammer, B.; Hansen, L. B.; Nørskov, J. K., Improved Adsorption Energetics within Density-Functional Theory Using Revised Perdew-Burke-Ernzerhof Functionals. Phys. Rev. B 1999, 59, 7413.

37. Blöchl, P. E., Projector Augmented-Wave Method. Phys. Rev. B 1994, 50, 17953.

38. Blöchl, P. E.; Jepsen, O.; Andersen, O.K. Improved Tetrahedron Method for BrillouinZone Integrations. Phys. Rev. B 1994, 49, 16223.

39. Monkhorst, H. J.; Pack, J. D., Special Points for Brillouin-Zone Integrations. Phys. Rev. $B$ 1976, 13, 5188 .

40. Momma K.; Izumi, F., VESTA 3 for Three-dimensional Visualization of Crystal, Volumetric and Morphology Data. J. Appl. Crystallogr. 2011, 44, 1272.

41. Kostorz, G., From CIF to Virtual Morphology Using the WinXMorph Program. J. Appl. Crystallogr. 2007, 40, 382.

42. Ruvireta, J.; Vega, L.; Viñes, F. Cohesion and Coordination Effects on Transition Metal Surface Energies. Surf. Sci. 2017, 664, 45-49.

43. Comsa, G.; Gelberg, A.; Iosifescu, B. Temperature Dependence of the Work Function of Metals (Mo,Ni). Phys. Rev. 1961, 122, 1091. 
44. Kiejna, A.; Wojciechowski, K. F.; Zebrowksi, J. The Temperature Dependence of Metal Work Functions. J. Phys. F: Met. Phys. 1979, 9, 1361-1366.

45. Curtiss, L. A.; Raghavachari, K.; Trucks, G. W.; Pople, J. A. Gaussian-2 Theory for Molecular Energies of First- and Second-row Compound. J. Chem. Phys. 1991, 94, 7221.

46. Sun, J.; Ruzsinszky, A.; Perdew, J. P. Strongly Constrained and Appropriately Normed Semilocal Density Functional. Phys. Rev. Lett. 2015, 115, 036402.

47. Peng, H.; Yang, Z. H.; Perdew, J. P.; Sun, J. Versatile van der Waals Density Functional Based on a Meta-generalized Gradient Approximation. Phys. Rev. X 2016, 6, 041005.

48. Patra, A.; Bates, J. E.; Sun, J.; Perdew, J. P. Properties of Real Metallic Surfaces: Effects of Density Functional Semilocality and van der Waals Nonlocality. Proc. Natl. Acad. Sci. 2017, 114, E9188-E9196. 